\title{
56. ON THE DISTRIBUTION OF SPACE VELOCITIES OF OB STARS
}

\author{
L. V. MIRZOYAN and M. A. MNATSAKANIAN \\ Bjurakan Astrophysical Observatory, Erevan, Armenia, U.S.S.R.
}

OB stars, as the most typical members of O-associations, are of great interest for the problems of stellar evolution. Their concentration in spiral arms indicates the importance of the $\mathrm{O}$-associations for the formation of the spiral arm population in galaxies.

In this report we present briefly the results of a study of the space velocities of O-B1 stars in stellar associations which can give some information on the internal motions in the spiral arms.

We have considered the so called synthetic association formed by superposing the subsystems of O-Bl stars around the nuclei of all known O-associations (Mirzoyan, 1961). If we assume that the associations are expanding, as has been predicted by Ambartsumian $(1949,1954)$ then we have to expect an increase of the mean velocity of expansion $V$ with the distance $r$ from the centre of the synthetic association as a result of the large dispersion of runaway velocities and non-simultaneous formation of stars in associations. We can assume as well that the intensity of star formation in the synthetic association, that is in the observed volume of the Galaxy during the last tens of millions of years has remained almost constant for all O-associations (Mirzoyan, 1965).

The first rough estimates of the velocities of O-B1 stars situated at different distances from the centre of the synthetic association confirmed the expected increase of the mean velocity with distance from the centre (Mirzoyan, 1961). Here we have applied a more correct method for the derivation of the function $V(r)$.

The general solution of the problem is presented by a formula which needs the use of the differentials of the observed distribution functions. Therefore the accuracy given by this formula is not high enough.

However, the problem becomes much simpler when we study the unknown function $V(r)$ only quantitatively. In this case one may express the mean values of $\bar{V}$ and $\bar{r}$ for the stars situated in plane-parallel layers in the projection of the association by two functions which can be determined from the observations: the number of stars and the sum of absolute radial velocities of stars in a circle of a given radius around the centre. Changing the widths and the positions of the layers in the association one may determine $\bar{V}$ for different $\bar{r}$ and, thus, the function $\bar{V}(\bar{r})$.

For the determination of $\bar{V}(\vec{r})$ we have used the radial velocities of 290 O-B1 stars from the Wilson (1953) catalogue. The distribution of these stars around the nuclei of stellar associations has been determined on the basis of their distances from the nearest nuclei. The distances of O-B1 stars have been taken from Hiltner's 


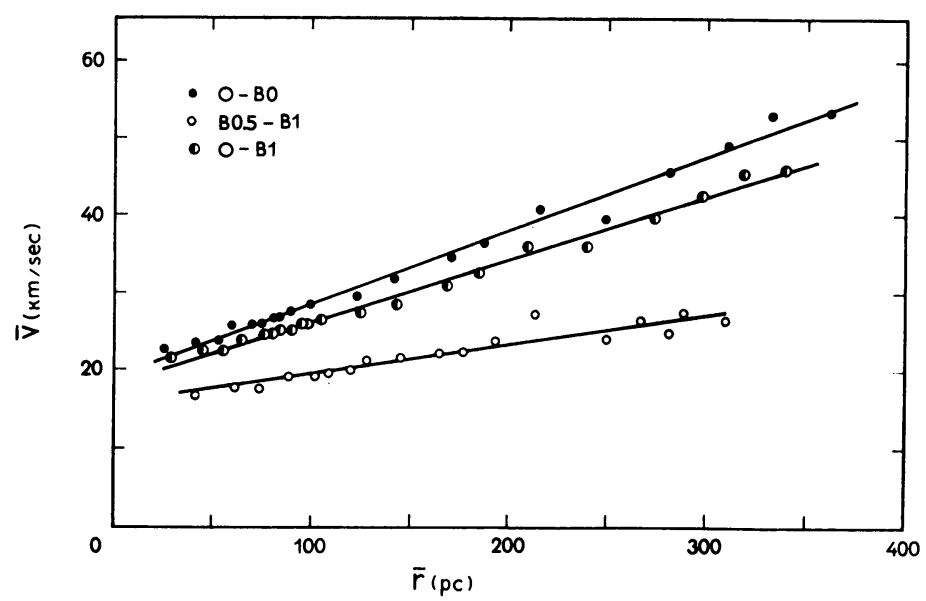

Fig. 1. The dependence $\bar{V}(\bar{r})$ in the synthetic association: for O-B0 (full dots), B0.5-B1 (open circles) and $\mathrm{O}-\mathrm{B} 1$ (semifilled circles) stars. The lines are the solutions by the least-square method.

(1955) list. For the stellar associations the data of Ruprecht's (1966) catalogue have been used.

The results of calculations for O-B0 stars (222), B0.5-B1 stars (68) and for all O-B1 stars (290) are presented in Figure 1, where the lines are obtained by the leastsquare method.

It is well seen in Figure 1, that the dependence of mean velocity from the distance to the centre is almost linear in all three cases.

It can be shown, that in this particular case, when the function $\bar{V}(\bar{r})$ is linear, the unknown function $V(r)$ must be linear as well. Moreover, in this case both functions must be identically equal: $V(r) \equiv \bar{V}(\bar{r})$.

Thus the analysis of the distribution of velocities of O-B1 stars based on the radial velocities and the distribution of these stars around the nuclei of associations confirm in full the conclusion concerning the increase of the mean velocities of stars with their distances from the giving birth nuclei (Mirzoyan, 1961). This fact is a strong evidence in support of the expansion of stellar associations.

Therefore the existing distribution of O-B stars around the nuclei of stellar associations and in the spiral arms of our Galaxy is caused by the intensity of star formation during the life-time of these systems and by the process of star aging with running away from the parent nuclei (Mirzoyan, 1965).

\section{References}

Ambartsumian, V. A.: 1949, Astron. Zh. 26, 3.

Ambartsumian, V. A.: 1954, Trans. IAU 8, 665.

Hiltner, W. A.: 1955, Astrophys. J. Suppl. 2, 389.

Mirzoyan, L. V.: 1961, Soobshch. Bjurak. Obs. 29, 81.

Mirzoyan, L. V.: 1965, Astrofiz. 1, $109=1965$, Astrophys. $1,70$.

Ruprecht, J.: 1966, Trans. IAU 13A, 830.

Wilson, R. E.: 1953, General Catalogue of Stellar Radial Velocities, Washington. 